\title{
Does conventional laser photocoagulation still have a place in the treatment of diabetic macular edema?
}

\author{
Belma Kayhan*, Engin Burumcek** \\ *Department of Ophthalmology, University of Health Sciences, Sultan 2. Abdulhamid Han \\ Training and Research Hospital, Istanbul, Turkey \\ **Department of Ophthalmology, Osmanoglu Hospital, Istanbul, Turkey
}

Correspondence to: Belma Kayhan, MD,

Department of Ophthalmology, University of Health Sciences, Sultan 2. Abdulhamid Han

Training and Research Hospital, Istanbul,

Acibadem, Almondhill Sitesi, B 15 Blok, D:8, Uskudar, 34660, Istanbul, Turkey,

Mobile phone: +90 5422927 804, E-mail: drbelmakayhan@gmail.com,

ORCID: 0000-0003-0748-6691

Accepted: May 9th, 2021

\begin{abstract}
Objective: The study aimed to investigate the long-term efficacy of conventional laser photocoagulation in the treatment of diabetic macular edema.

Methods: In this retrospective study, the records of patients presented with diabetic macular edema (DME) and non-proliferative diabetic retinopathy were reviewed. DME defined as clinically significant macular edema was treated by using argon green or yellow dye laser with focal, grid, and modified grid techniques according to Early Treatment Diabetic Retinopathy Study parameters. Best-corrected visual acuity (BCVA) was measured. BCVA change after the treatment and its relationship with other factors were evaluated.

Results: The study included 133 eyes of 81 patients. The mean follow-up was 28.26 months. BCVA demonstrated the increase of 2 lines or more in $20.7 \%$ of the eyes, stabilization within 2 lines in $60.7 \%$ of the eyes, and loss of 2 lines or more in $18.3 \%$ of the eyes. The eyes with baseline BCVA lower than or equal to 0.50 showed a statistically significant increase $(p=0.001)$ whereas the eyes with baseline BCVA of more than 0.50 did not show a statistically significant change $(\mathrm{p}=0.070)$ after laser photocoagulation treatment.

Conclusions: Conventional laser photocoagulation is an effective treatment in diabetic macular edema including center-involved type and stabilizes visual acuity in the majority of the patients. Improvement in BCVA is significant in the group with lower baseline BCVA.

Keywords: laser photocoagulation, diabetic macular edema, focal laser, grid laser

Abbreviations: $\mathrm{DM}=$ diabetes mellitus, $\mathrm{DME}=$ diabetic macular edema, ETDRS = early treatment diabetic retinopathy study, CSME = clinically significant macular edema, CLP = conventional laser photocoagulation, VEGF = vascular endothelial growth factor, BCVA = best-corrected visual acuity, ANOVA = analysis of variance, $\mathrm{VA}=$ visual acuity
\end{abstract}

\section{Introduction}

Diabetes mellitus (DM) has an increasing prevalence all over the world. The Global Burden of Disease Study 2017 states that the incidence and prevalence of DM were 22.9 million, and 476.0 million, respectively in 2017, with a projection to 26.6 million and 570.9 million in 2025 [1]. The overall prevalence of diabetic macular edema (DME) is about $7 \%$ and it means that at least 40 million people will probably suffer from DME in the near future [2].
DME is the most common cause of acquired visual loss in this population and hence, treatment of DME is of great importance. After Early Treatment Diabetic Retinopathy Study (ETDRS) defined clinically significant macular edema (CSME) and its treatment with laser photocoagulation [3,4], conventional laser photocoagulation (CLP) has become the only treatment option. Currently, anti-vascular endothelial growth factor (anti-VEGF) injections are the most preferred treatments in DME. Other new treatment modalities, such as intravitreal corticosteroid 
injections or implants, subthreshold micropulse lasers have also been introduced for DME treatment. Although these new treatments became increasingly widespread all over the world, they have some disadvantages compared with CLP. These treatments require several injections and/or treatment sessions and control examinations. They are expensive and necessitate good patient attendance. Moreover, some eyes with DME can be refractory to these treatments [5].

In this study, we aimed to investigate the longterm effectiveness and safety of CLP and to evaluate its potential to be a sustainable option in the treatment of DME.

\section{Materials and methods}

In this retrospective study, medical records of diabetic patients referred to the retina department were reviewed. This study was conducted according to the Declaration of Helsinki and was approved by the Institutional Review Board (Approval No: 48670771-903.99). The patients who had nonproliferative diabetic retinopathy and DME and who were treatment-naive were included in the study. The patients with proliferative diabetic retinopathy or who progressed to the proliferative stage during treatments were excluded. Other exclusion criteria were history of cataract surgery for the last one year, history of another intraocular surgery, glaucoma or other ocular diseases including intraocular inflammations, eyes with nonperfusion areas within the perifoveal capillary network. Patients with hemoglobin A1c > $64 \mathrm{mmol} / \mathrm{mol}(>8 \%$ ) at presentation were defined as having uncontrolled DM and were excluded from the study. The presence of diastolic blood pressure over $100 \mathrm{mmHg}$ and/or chronic kidney failure were other reasons for exclusion.

Best-corrected visual acuity (BCVA) was measured by the Snellen visual acuity chart. Routine, complete ophthalmological examination, colored fundus photography and fundus fluorescein angiography were performed at baseline and followup examinations. Follow-ups took place 3 weeks after the 1st treatment, every 3 months for the first year, and every 6 months after the first year.

Eyes with focal or diffuse edema defined as CSME were treated with argon green $(514 \mathrm{~nm})$ or yellow dye $(570 \mathrm{~nm})$ laser randomly. CSME was defined accordingly to ETDRS protocol as retinal thickening at or within $500 \mu \mathrm{m}$ of the center of the macula; hard exudates at or within $500 \mu \mathrm{m}$ of the center of the macula, if associated with adjacent retinal thickening; or a zone or zones of retinal thickening one disc area in size, at least part of which was within one disc diameter of the center of the macula [3]. Diffuse macular edema was classified into cystoid and noncystoid types. Successive treatments were performed with the same wavelength laser as the first treatment of the eye. Topical anesthesia was applied to all patients. Grid or modified grid laser photocoagulation was performed in the treatment of diffuse macular edema. In grid photocoagulation, $200 \mu \mathrm{m}$ and 0.1 -sec laser spots were used on the thickened retinal area by forming mild severity of laser burns and leaving one spot interval among laser spots. In focal treatment, laser spots of $100 \mu \mathrm{m}$ and $0.1 \mathrm{sec}$ were applied on every microaneurysm by obtaining slight whitening at the level of retinal pigment epithelium, subsequently, whitening, or darkening of the microaneurysm was observed with the application of additional 50 or $100 \mu \mathrm{m}$ spots. In modified grid treatment, focal treatment was added to grid treatment. Persistent retinal thickening or new lesions detected during follow-up examinations were treated with additional laser photocoagulation.

\section{Statistical Analysis}

SPSS for Windows was used for statistical analysis. The effect of laser treatment on BCVA, correlations with variables related to patients and treatment were analyzed with this statistics program. Students $t$, Mann Whitney U, paired $t$, and ANOVA tests were used for comparisons. $\mathrm{p}<0.05$ was accepted for statistical significance.

\section{Results}

The study comprised 133 eyes of 81 patients. One eye of 29 patients and both eyes of 52 patients were included. Forty (49.4\%) patients were females, 41 (50.6\%) patients were males. The mean duration of DM was 10.88 years (ranged from 6 months to 29 years). The mean follow-up was 28.26 months (ranged from 3 to 100 months). Yellow dye laser was applied to 17 eyes and argon green laser was applied to 116 eyes. While 112 eyes (84.2\%) required treatment once, 17 eyes (12.8\%) were treated twice and four eyes $(3 \%)$ were treated three times (mean $1.19 \pm 0.46$ ). Table 1 shows the number of eyes and applied laser treatment techniques.

Table 1. Applied laser treatment techniques and the number of the eyes

\begin{tabular}{ll} 
Treatment Technique & Number of Eyes \\
\hline Only Focal & 35 \\
Only Grid & 35 \\
Only Modified Grid & 42 \\
Focal + Focal & 2 \\
Focal + Grid & 3 \\
Focal + Modified Grid & 1 \\
Grid + Grid & 4 \\
Grid + Modified Grid & 4
\end{tabular}




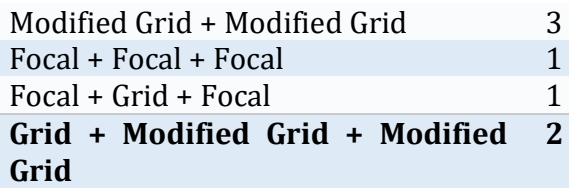

BCVA change after treatment was analyzed and three eyes with BCVA lower than 0.10 were exempted from the analysis. Patients were divided into two groups according to baseline BCVA lower than or equal to 0.50 or more than 0.50 . The first group with lower BCVA responded to the treatment better and BCVA after the laser treatment showed a statistically significant increase, whereas the second group with BCVA of more than 0.50 did not show a statistically significant change (Table 2).

Table 2. Change in BCVA according to baseline BCVA level

$\begin{array}{ll}\text { Baseline BCVA }< & \text { Baseline BCVA }> \\ =0.50 & 0.50\end{array}$

$\begin{array}{lll}\begin{array}{l}\text { Baseline } \\ \begin{array}{l}\text { BCVA } \\ \text { (mean } \pm \text { SD) }\end{array}\end{array} & 0.36 \pm 0.01 & 0.78 \pm 0.13 \\ \begin{array}{l}\text { Last BCVA } \\ \text { (mean } \pm \text { SD) }\end{array} & 0.46 \pm 0.21 & 0.74 \pm 0.23 \\ & \mathbf{p}=\mathbf{0 . 0 0 1} & \mathrm{p}=0.070\end{array}$

*BCVA is expressed in decimal system

$B C V A=$ best-corrected visual acuity, $S D=$ standard deviation

When BCVA change of all eyes in the study was evaluated, the mean baseline BCVA was $0.63 \pm 0.24$ and the mean BCVA at the last visit was $0.65 \pm 0.26$ and the difference was not statistically significant $(\mathrm{p}>$ $0.05)$. At the last follow-up, CLP treatment preserved BCVA in 79 eyes $(60.7 \%)$ and improved more than 2 lines in 27 eyes (20.7\%) (Fig. 1).

BCVA change was analyzed according to the applied laser types. BCVA change in 113 eyes treated with argon green laser did not show a statistical significance (Baseline BCVA, $0.64 \pm 0.23$; last BCVA, $0.64 \pm 0.26 ; p=0.94)$. Seventeen eyes treated with yellow dye laser showed statistically significant improvement in BCVA after treatment (Baseline BCVA, $6.23 \pm 0.28$; last BCVA, $0.70 \pm 0.25$; $\mathrm{p}=0.03$ ).

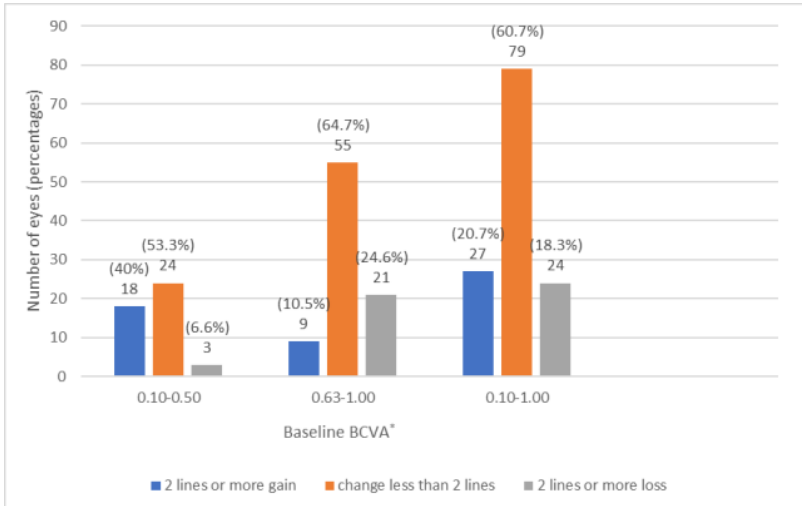

Fig. 1 BCVA change at the last follow-up examination. In the first group with baseline best-corrected visual acuity (BCVA) between 0.10 and 0.50 , conventional laser photocoagulation (CLP) stabilized BCVA within 2 lines in 24 eyes (53.3\%), increased more than 2 lines in 18 eyes (40\%), and decreased in three eyes (6.6\%).

In the second group with baseline BCVA between 0.63 1.00 , CLP stabilized BCVA within 2 lines in 55 eyes $(64.7 \%)$, increased more than 2 lines in nine eyes $(10.5 \%)$ and decreased more than 2 lines in 21 eyes (24.6\%).In total analysis, 27 eyes $(20.7 \%)$ showed BCVA increase of 2 lines or more, 79 eyes $(60.7 \%)$ stabilization within 2 lines, and 24 eyes (18.3\%) loss of 2 lines or more, respectively.

*BCVA = best-corrected visual acuity

Eyes with focal macular edema had the highest baseline BCVA, followed by diffuse non-cystoid macular edema and cystoid macular edema, respectively. However, BCVA change after laser treatment did not demonstrate a statistically significant difference in any type of DME (Table 3).

Table 3. BCVA change after laser photocoagulation treatment according to types of DME

\begin{tabular}{|c|c|c|c|c|c|}
\hline & $\begin{array}{l}\text { Focal DME } \\
(n=40)\end{array}$ & $\begin{array}{l}\text { Non-cystoid diffuse } \\
\text { DME }(n=65)\end{array}$ & $\begin{array}{l}\text { Cystoid diffuse } \\
\text { DME }(n=25)\end{array}$ & $\mathbf{F}$ & $\mathbf{p}$ \\
\hline $\begin{array}{l}\text { Baseline BCVA * } \\
(\text { mean } \pm \text { SD) }\end{array}$ & $0.70 \pm 0.23$ & $0.66 \pm 0.22$ & $0.46 \pm 0.21$ & 9.09 & 0.0002 \\
\hline \multirow{2}{*}{$\begin{array}{l}\text { Last BCVA * } \\
(\text { mean } \pm \text { SD) }\end{array}$} & $0.70 \pm 0.25$ & $0.68 \pm 0.24$ & $0.48 \pm 0.26$ & 6.87 & 0.0015 \\
\hline & $\mathrm{p}=0.857$ & $\mathrm{p}=0.534$ & $\mathrm{p}=0.824$ & & \\
\hline
\end{tabular}

*BCVA is expressed in decimal system

$B C V A=$ best -corrected visual acuity, $D M E$ = diabetic macular edema, $S D=$ standard deviation

BCVA changes were also evaluated according to type and duration of $\mathrm{DM}$ and received systemic treatments (Table 4). While the systemic treatment type and duration of diabetes did not show a statistically significant effect on BCVA change, BCVA in patients with DM type 1 revealed a statistically significant increase after laser photocoagulation treatment. 
Table 4. BCVA changes according to systemic treatment type, duration, and type of DM

\begin{tabular}{|c|c|c|c|c|c|c|}
\hline & $\begin{array}{l}\text { Insulin } \\
(n=98)\end{array}$ & $\begin{array}{l}\text { OAD } \\
(n=32)\end{array}$ & $\begin{array}{l}\mathrm{DM}<=10 y \\
(n=71)\end{array}$ & $\begin{array}{l}D M>10 y \\
(n=59)\end{array}$ & $\begin{array}{l}\text { Type I DM } \\
(n=7)\end{array}$ & $\begin{array}{l}\text { Type II DM } \\
(n=123)\end{array}$ \\
\hline $\begin{array}{l}\text { Baseline BCVA * } \\
(\text { mean } \pm \text { SD) }\end{array}$ & $0.64 \pm 0.24$ & $0.61 \pm 0.22$ & $0.62 \pm 0.24$ & $0.65 \pm 0.23$ & $0.60 \pm 0.33$ & $0.64 \pm 0.23$ \\
\hline \multirow[t]{2}{*}{$\begin{array}{l}\text { Last BCVA * } \\
(\text { mean } \pm \text { SD) }\end{array}$} & $0.66 \pm 0.24$ & $0.59 \pm 0.31$ & $0.64 \pm 0.26$ & $0.65 \pm 0.26$ & $0.72 \pm 0.24$ & $0.64 \pm 0.26$ \\
\hline & $p=0.206$ & $p=0.647$ & $\mathrm{p}=0.320$ & $\mathrm{p}=0.852$ & $p=0.022$ & $\mathrm{p}=0.814$ \\
\hline
\end{tabular}

*BCVA is expressed in decimal system

$B C V A=$ best -corrected visual acuity, $D M=$ diabetes mellitus, $O A D=$ oral antidiabetic drugs, $S D=$ standard deviation, $y=y e a r s$

In one eye with BCVA of 0.80 before treatment, exudative plaque arose after 33 months of the treatment, and BCVA decreased to $0.5 \mathrm{~m}$ finger counting level. This patient had the diffuse type of macular edema and was treated by modified grid laser technique twice with an 18 months-interval. No other adverse effect was observed in the study group.

\section{Discussion}

Currently, anti-VEGF intravitreal injections are the first-line treatment in DME. Steroid injections, subthreshold micropulse lasers have also been introduced as alternative treatments [6]. Albeit all these new treatments have started to predominate in clinical practice, CLP could not be replaced entirely in the treatment of DME and it is used as a single treatment or a part of combination therapy. In the current study, we reviewed the outcomes of CLP in the treatment of DME when none of the currently used other options for treatment were present. Our study showed a BCVA increase of 2 lines or more in about $21 \%$ of the eyes and stabilization within 2 lines in about $61 \%$ of the eyes. Laser photocoagulation demonstrated very good efficacy in the preservation of visual acuity (VA) in CSME including centerinvolved types in more than $80 \%$ of the eyes. These outcomes were in line with ETDRS, which reported that immediate laser photocoagulation in eyes with CSME could reduce the percentage of eyes with moderate visual loss by at least $50 \%$ and VA improved in $16 \%$, remained unchanged in $77 \%$, and worsened in $7 \%$ of the treated eyes [3]. In a study by Lee et al., laser photocoagulation improved VA in $14.5 \%$, unchanged in $60.9 \%$ of the eyes with diffuse DME [7]. Similarly, Scott et al. evaluated the effect of focal/ grid photocoagulation on VA in eyes with noncenter involved CSME and $75 \%$ of the patients preserved $20 / 25$ or better visual acuity at one year after treatment [8].

Several studies reported that baseline BCVA was a strong predictor of BCVA changes after laser photocoagulation in DME and patients with poorer VA achieved greater gains than those with better baseline vision $[\mathbf{9 , 1 0}]$. Comparably, improvement in BCVA was significant and better in the group with lower baseline BCVA in our study.

We compared VA change among groups classified according to types of edema; focal, non-cystoid diffuse edema, and cystoid diffuse edema and the results did not reveal any effect of edema type on VA change although baseline VA demonstrated a statistically significant difference between groups, the best with focal DME and the worst with cystoid diffuse DME.

In the last decade, new laser models called subthreshold micropulse lasers have been introduced for the treatment of diffuse DME. Those new lasers aimed to deliver less thermal energy and claimed to create less photothermal damage to the neurosensory and inner retina than continuous-wave conventional lasers and hence, to induce less undesirable side effects such as visual field defects, epiretinal fibrosis, and choroidal neovascularization in the area of the laser scar. Although some studies reported better BCVA with subthreshold micropulse lasers, several studies comparing with conventional lasers stated a clinically insignificant difference in improving VA [1114].

With the presentation of the first anti-VEGF pegaptanib for the treatment of DME, bevacizumab, ranibizumab, and aflibercept were introduced into the clinical practice sequentially. Several studies showed improvement in VA in center-involved DME [15-17]. However, Baker et al. compared the outcomes of initial management with aflibercept or with laser treatment or observation and given aflibercept only if visual acuity worsened in eyes with center-involved DME and good VA. They found no significant difference in vision loss at 2 years among three groups and thus no superiority of aflibercept injections over laser photocoagulation [18]. AntiVEGF treatment necessitates several monthly injections to achieve desirable results during treatment of DME and causes treatment burden both financially and related to patient attendance [16]. Moreover, every injection may carry some risks such as endophthalmitis, cerebrovascular accidents even if they are seen very rarely $[\mathbf{1 9 , 2 0}]$. 


\section{Conclusion}

In conclusion, our study demonstrated that CLP stabilized or improved visual acuity in $81 \%$ of the eyes for a long-term follow-up. Although anti-VEGF injections are the main treatment modality in centerinvolved DME and additional treatments such as corticosteroid injections and subthreshold micropulse lasers have been presented as alternative treatments, CLP still has a role in the treatment of DME because of its good outcomes, low cost and fewer treatment sessions for patients who have difficulties in attending appointments. The limitation of our study is its retrospective design and lack of control groups.

\section{Conflict of Interest statement}

The authors state no conflict of interest.

\section{Informed Consent and Human and Animal Rights statement}

Informed consent has been obtained from all individuals included in this study.

\section{Authorization for the use of human subjects}

Ethical approval: The research related to human use complies with all the relevant national regulations, institutional policies, is in accordance with the tenets of the Helsinki Declaration, and has been approved by the institutional review board of the University of Health Sciences, Sultan 2. Abdulhamid Han Training and Research Hospital, Istanbul, Turkey (Approval No: 48670771-903.99).

\section{Acknowledgments}

None.

\section{Sources of Funding}

This study received no public or private support.

\section{Disclosures}

None.

\section{References}

1. Lin X, Xu Y, Pan X, Xu J, Ding Y, Sun X, Song X, Ren Y, Shan PF. Global, regional, and national burden and trend of diabetes in 195 countries and territories: an analysis from 1990 to 2025. Sci Rep. 2020; 10(1):14790.

2. Yau JW, Rogers SL, Kawasaki R, Lamoureux EL, Kowalski JW, Bek T, Chen SJ, Dekker JM, Fletcher A, Grauslund J, Haffner S, Hamman RF, Ikram MK, Kayama T, Klein BE, Klein R, Krishnaiah S, Mayurasakorn K, O'Hare JP, Orchard TJ, Porta M, Rema M, Roy MS, Sharma T, Shaw J, Taylor H, Tielsch JM, Varma R, Wang JJ, Wang N, West S, Xu L, Yasuda M, Zhang X, Mitchell P, Wong TY. Meta-Analysis for Eye Disease (METAEYE) Study Group. Global prevalence and major risk factors of diabetic retinopathy. Diabetes Care. 2012; 35(3):556-564.

3. Early Treatment Diabetic Retinopathy Study Research Group. Photocoagulation for diabetic macular edema. Early
Treatment Diabetic Retinopathy Study report number 1. Arch Ophthalmol. 1985; 103(12):1796-1806.

4. Early Treatment Diabetic Retinopathy Study Research Group. Focal photocoagulation treatment of diabetic macular edema. Relationship of treatment effect to fluorescein angiographic and other retinal characteristics at baseline: ETDRS report no. 19. Arch Ophthalmol. 1995; 113(9):11441155.

5. Bressler SB, Odia I, Glassman AR, Danis RP, Grover S, Hampton GR, Jampol LM, Maguire MG, Melia M. Changes in diabetic retinopathy severity when treating diabetic macular edema with ranibizumab: DRCR.net Protocol I 5-Year Report. Retina. 2018; 38(10):1896-1904.

6. Jorge EC, Jorge EN, Botelho M, Farat JG, Virgili G, El Dib R. Monotherapy laser photocoagulation for diabetic macular oedema. Cochrane Database Syst Rev. 2018; 10(10):CD010859.

7. Lee CM, Olk RJ. Modified grid laser photocoagulation for diffuse diabetic macular edema. Long-term visual results. Ophthalmology. 1991; 98(10):1594-1602.

8. Scott IU, Danis RP, Bressler SB, Bressler NM, Browning DJ, Qin H. Effect of focal/grid photocoagulation on visual acuity and retinal thickening in eyes with non-center-involved diabetic macular edema. Retina. 2009; 29(5):613-617.

9. Aiello LP, Edwards AR, Beck RW, Bressler NM, Davis MD, Ferris F, Glassman AR, Ip MS, Miller KM. Diabetic Retinopathy Clinical Research Network. Factors associated with improvement and worsening of visual acuity 2 years after focal/grid photocoagulation for diabetic macular edema. Ophthalmology. 2010; 117(5):946-953.

10. Mitchell P, Bandello F, Schmidt-Erfurth U Lang GE, Massin P, Schlingemann RO, Sutter F, Simader C, Burian G, Gerstner O, Weichselberger A. RESTORE study group. The RESTORE study: ranibizumab monotherapy or combined with laser versus laser monotherapy for diabetic macular edema. Ophthalmology. 2011; 118(4):615-625.

11. Scholz P, Altay L, Fauser S. A review of subthreshold micropulse laser for treatment of macular disorders. Adv Ther. 2017; 34(7):1528-1555.

12. Ohkoshi K, Yamaguchi T. Subthreshold micropulse diode laser photocoagulation for diabetic macular edema in Japanese patients. Am J Ophthalmol. 2010; 149(1):133-139.

13. Chen G, Tzekov R, Li W, Jiang F, Mao S, Tong Y. Subthreshold micropulse diode laser versus conventional laser photocoagulation for diabetic macular edema: a metaanalysis of randomized controlled Trials. Retina. 2016; 36(11):2059-2065.

14. Wu Y, Ai P, Ai Z, Xu G. Subthreshold diode micropulse laser versus conventional laser photocoagulation monotherapy or combined with anti-VEGF therapy for diabetic macular edema: A Bayesian network meta-analysis. Biomed Pharmacother. 2018; 97(1):293-299.

15. Wells JA, Glassman AR, Ayala AR, Jampol LM, Aiello LP, Antoszyk AN, Arnold-Bush B, Baker CW, Bressler NM, Browning DJ, Elman MJ, Ferris FL, Friedman SM, Melia M, Pieramici DJ, Sun JK, Beck RW. Aflibercept, bevacizumab, or ranibizumab for diabetic macular edema. N Engl J Med. 2015; 372(13):1193-1203.

16. Wells JA, Glassman AR, Ayala AR, Jampol LM, Bressler NM, Bressler SB, Brucker AJ, Ferris FL, Hampton GR, Jhaveri C, Melia M, Beck RW. Diabetic Retinopathy Clinical Research Network. Aflibercept, bevacizumab, or ranibizumab for diabetic macular edema: two-year results from a comparative effectiveness randomized clinical trial. Ophthalmology. 2016; 123(6):1351-1359.

17. Cai S, Bressler NM. Aflibercept, bevacizumab or ranibizumab for diabetic macular oedema: recent clinically relevant findings from DRCR.net Protocol T. Curr Opin Ophthalmol. 2017; 28(6):636-643.

18. Baker CW, Glassman AR, Beaulieu WT, Antoszyk AN, Browning DJ, Chalam KV, Grover S, Jampol LM, Jhaveri CD, 
Melia M, Stockdale CR, Martin DF, Sun JK. DRCR Retina Network. Effect of initial management with aflibercept vs laser photocoagulation vs observation on vision loss among patients with diabetic macular edema involving the center of the macula and good visual acuity: A Randomized Clinical Trial. JAMA. 2019; 321(19):1880-1894.

19. Avery RL, Gordon GM. Systemic safety of prolonged monthly anti-vascular endothelial growth factor therapy for diabetic macular edema: A Systematic review and metaanalysis. JAMA Ophthalmol. 2016; 134(1):21-29.

20. Porta M, Striglia E. Intravitreal anti-VEGF agents and cardiovascular risk. Intern Emerg Med. 2020; 15(2):199-210. 